\title{
A Research on the Level of the Coordinated Development in the Yangtze River Delta
}

\author{
Baoqian Wang, Jingjing Li, Cheng Wang \\ Business School, Hohai University, Nanjing, China \\ Email: ouyumu1989@126.com
}

Received 5 March 2014; revised 28 March 2014; accepted 8 April 2014

Copyright (C) 2014 by authors and Scientific Research Publishing Inc.

This work is licensed under the Creative Commons Attribution International License (CC BY).

http://creativecommons.org/licenses/by/4.0/

(c) (i) Open Access

\begin{abstract}
By using the Factor Analysis, this paper estimates the level of coordinated development among the 16 cities in Yangtze River Delta. The absolute value of the Moran I index from 2007 to 2011 is 0.3759 , $0.3307,0.3496,0.2608$ and 0.1547 respectively, indicating a relatively close spatial relationship among city growth poles. The database taken into the empirical analysis meets the request of the KMO and Bartlett tests. The empirical results show that the Skewness value of the economic, social and environmental value is $1.821,-0.096,0.743$, and 1.373 respectively and with a correspondent Kurtosis value of $3.618,-0.722,-0.774$ and 1.607 . It is revealed that the diversity of the comprehensive index value is serious, which deprived mainly from the economic difference. On the other hand, the diversity of the society and environment is benefit for the coordinated development among the cities in Yangtze River Delta. In the future development, the 16 cities should seek for individualized development with its own resource basement. While for the social and environmental development, the policy integration of the society, environment and human resource should be realized to promote the mobility of the resources hence to raise the level of coordinated development.
\end{abstract}

\section{Keywords}

Growth Pole, Yangtze River Delta, Level of Coordinated Development

\section{Introduction}

Since China adopts the policy of reform and opening up, Chinese government has made the strategy of giving priority to the development of the coastal areas, which successfully cultivates China's three main regional economic growth poles, namely the Yangtze River Delta, the Pearl River Delta and the Bohai Economic Rim.

Among the three main regional economic growth poles, the Yangtze River Delta has become one vital engine of China's economy development. It created 19\% of China's total GDP, 22\% of China's total taxation revenue and $18.4 \%$ of China's total exports with only $1 \%$ of China's lands and $5.8 \%$ of the total population. By analyz- 
ing the level of the coordinated development among the internal cities of the Yangtze River Delta, this paper studies the methods to stimulate the coordinated development within this area (Figure 1).

\section{Literature Review}

The literatures about the coordinated development mainly contain the following three parts: the polarization process helps the development of the growth pole itself, the polarization process results in the diversity between surrounding areas, and finally how the regional economic system coordinates the development between the pole and surrounding area.

\subsection{Agglomeration Effects}

In 1950s, the French economist F. Perroux proposes that "both the Harold-Doha Model and the Neo-classical economic bear the deficiencies that the growth in those models is flat and regular". In fact, "the growth will never come up in every place" [1]. The growth will come up in growth poles in difference magnitude, and then such growth from the pole will spread out in various channels hence impose a final influence on total economy. G. Myrdal and A. O. Hirschman summarize the influence that the growth poles impose on the surrounding areas or the cities' interior places in two contrasting effects, namely the polarization effect and the diffusion effect. With the help of polarization effect, elements and resources will concentrate in the pole area, bringing the agglomeration effects and scale economy, hence stimulating the development of the economy.

\subsection{Larger Development Gaps}

By making use of the index of GDP per person, Tsui (1991) discovers that during 1952 and 1970, the development gap of China’s regional development is not serious, while during 1970 and 1985 such gap becomes larger

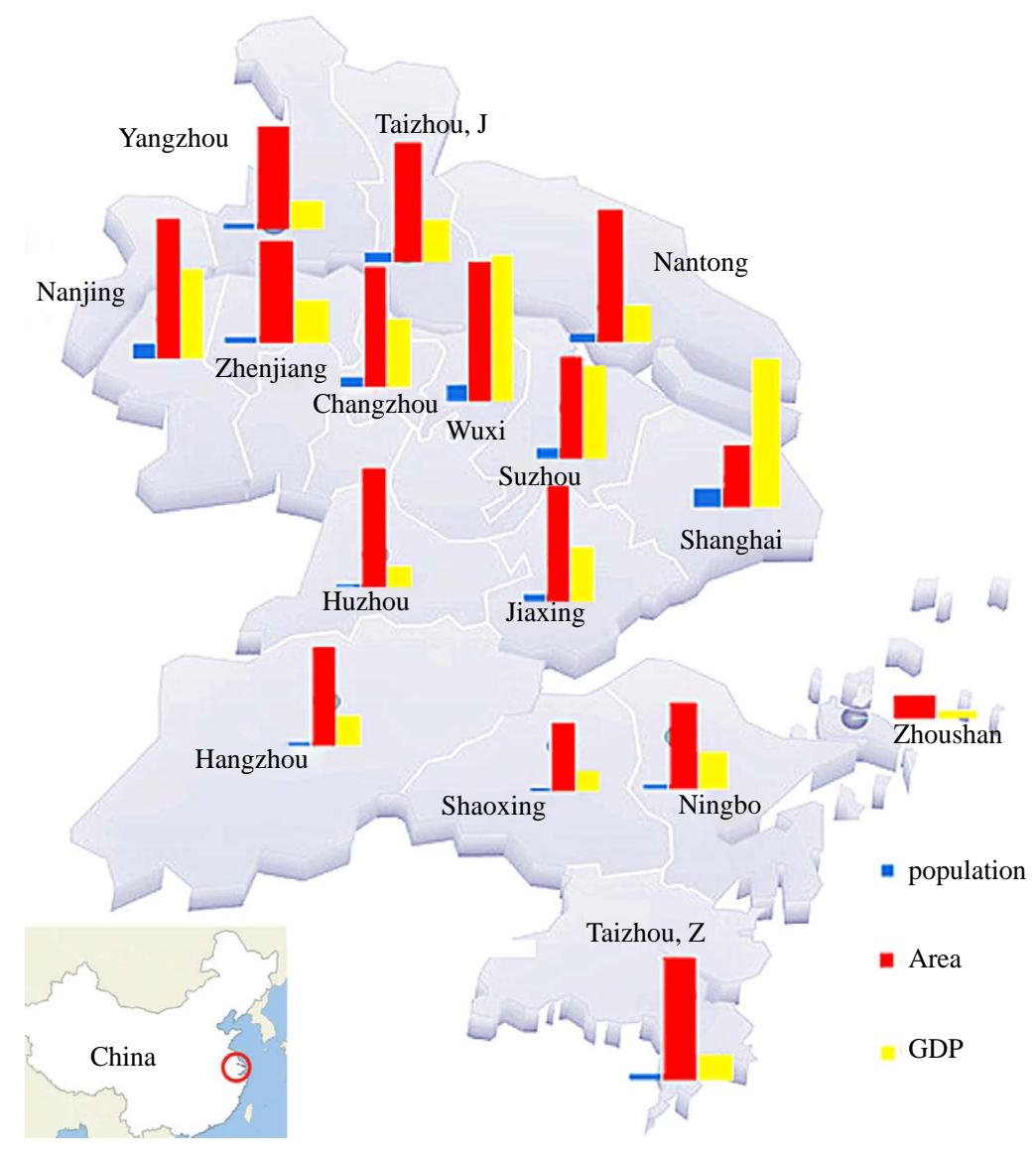

Figure 1. 16 city growth poles in the Yangtze River delta. 
than ever.

Yang Dali (1995) selects the statistics of China's rural economy from 1985 to 1990 so as to study the transition path of the development gap. The results show that in most provinces of China, the development gap indicates a continuing larger trend [2].

Based on the full part and partial spatial autocorrelation analysis of EDSA, Pu Yingxia, Ge Ying, Ma Ronghua, Huang Xingyuan and Ma Xiaodong (2005) make a research on the variation trend, characteristics and reasons of Jiangsu's rural space economy. The research results show that the space variation in Jiangsu's rural economy is in a narrower channel, which however, is not the symbol of coordinated development [3].

\subsection{The Coordinated Development between Poles and Surrounding Areas Because of the Diffusion Effect}

Liu Shuhui (2001) points out that the innovation technology can be spread and transmitted from higher gradient to lower ones. However, such spread should rely on an industrial abutment mechanism between various gradients [4].

He Youli and Zhang Renzhi (2007) research the problem of the regional development variation caused by the growth of poles, as well as the limited effect of the scale economy [5].

Zhang Zhaotong (2009) thinks that China's regional coordinated development should constantly promote the effect of diffusion and the industrial relationship to eliminate the obstacle of elements and resources flow, to clear up the viscosity of cooperate transition, and to increase the cooperates transmit strength and industrial illiquid [6].

Zhu Juncheng (2011) compares 16 cities in the Yangtze River Delta by making use of the index indicating the level of cities' economic development. Zhu makes the strategy that the 16cities in the Yangtze River Delta should cultivate multiple centers adhering to innovation development and cluster development in order to create a new urbanization way [7].

Deng Xiangrong and Liu Xuan (2007) make use of the Esteban-Ray index and impulse response function as tools to measure the agglomeration and diffusion effects in the Yangtze River Delta and the Bohai Economic $\operatorname{Rim}[8]$.

Considering all the literatures above, it is believed that dual effects work in the growth pole system. The growth pole brings in the development of the regional economy and on the other side, the growth pole results in the development variation between the poles and the surrounding areas. Through the empirical analysis, it is discovered that since China adopted the non-balanced development strategy, the development gap within China's internal economy is becoming larger. As a result, it is of vital importance that China should coordinate the development of the growth poles and the surrounding spaces.

\section{The Hypothesis Testing of the Coordination Degree of Growth Poles}

If the production elements could flow freely in the regional space, the dual effects of the growth pole will mean to be balanced. Because of the constraint of the diffusion effect, the agglomeration effect will never be too serious. As the exertion of the diffusion effect is closely related to the spatial autocorrelation, this paper assumes that if the element flow is without any barrier and the economy unit in space is auto correlated, the relationship among each unit will be coordinated, eliminating the randomness.

The hypothesis of the free element flow will be satisfied since the 16cities in the Yangtze River Delta is in the context of the domestic trade. Hence this paper will use Moran I index to analysis the autocorrelation among the 16cities in the Yangtze River Delta.

\subsection{Model Selection}

The Moran I index is a common tool analyzing the spatial autocorrelation, with entire Moran I index and part Moran I index involved. As this paper will simply testify the overall economic relationship among the 16 cities only, the paper will use entire Moran I to do the empirical analysis. If the value of the entire Moran I index is equal to 0 , each unit in space will not reflect the economic relationship, which means each unit shares random changes without any mutual influences. If the value of the entire Moran I index is not equal to 0 , each unit in space will reflect the economic relationship on the contrary. 
The model of the entire Moran I index is as follows:

$$
I=n \times \sum_{i=1}^{n} \sum_{j=1}^{n} w_{i j}\left(x_{i}-\bar{x}\right)\left(x_{j}-\bar{x}\right) / \sum_{i=1}^{n} \sum_{j=1}^{n} w_{i j} \sum_{i=1}^{n}\left(x_{i}-\bar{x}\right)^{2}
$$

I: the entire Moran I index;

$n$ : the number of the growth poles taking into the samples $(n=16)$;

$w_{i j}$ : the element in spatial weight coefficient matrix;

$x_{i}$ and $x_{j}$ : the GDP per person in the growth pole $i$ and the growth pole $j$;

$\bar{x}$ : the mean value of the GDP per person in the sample, $n=16$.

\subsection{The Determination Process of Spatial Weight Coefficient Matrix}

It is a key to determine the spatial weight coefficient matrix and its internal element before calculating the entire Moran I index. This paper adopts the principle of point adjacent to determine the spatial weight coefficient matrix. The model is as following:

$$
\begin{aligned}
W & =\left|\begin{array}{cccc}
w_{11} & w_{12} & \cdots & w_{1 n} \\
w_{21} & w_{22} & \cdots & w_{2 n} \\
\vdots & \vdots & \ddots & \vdots \\
w_{n 1} & w_{n 2} & \cdots & w_{n n}
\end{array}\right| \\
W_{i j} & = \begin{cases}1 & i \text { and } j \text { are adjacent } \\
0 & i \text { and } j \text { are not adjacent }\end{cases}
\end{aligned}
$$

The growth pole $i$ and $j$ are adjacent (1); the growth pole $i$ and $j$ are not adjacent (2).

Through the GeoDA software, according to the administrative divisions of the Yangtze River Delta, this paper could conveniently establish a spatial weight coefficient matrix.

After determining the analyzing methods, the spatial weight coefficient matrix and its elements of spatial autocorrelation, by making use of the GeoDA software, this paper could calculate the coefficient I of the 16 cities in the Yangtze River Delta from 2007 to 2011.

From the statistics in Table 1, from the year of 2007 to 2011, it is clear that in the Yangtze River Delta, there exists an economic spatial correlation. Such correlation becomes more tightened with the time flows, indicating a closer economic relationship among the 16 cities.

\section{The Selection and Establishment of the Index System}

\subsection{System Selection}

According to the principle of systemic, easily acquiring, quantifying, comparable, goal oriented and timeliness, this paper establishes the comprehensive index system measuring the level of regional economic development (Table 2).

\subsection{The Determination of the Indexes}

In this paper, the method of factor analysis will be used to build up the comprehensive index system measuring the level of urban growth level.

Firstly establish several new comprehensive variables by a linear combination of the initial indicators.

$$
\left\{\begin{array}{l}
Z_{1}=\alpha_{11} F_{1}+\alpha_{12} F_{2}+\cdots+\alpha_{1 n} F_{n}+c_{1} U_{1} \\
Z_{2}=\alpha_{21} F_{2}+\alpha_{22} F_{2}+\cdots+\alpha_{2 n} F_{n}+c_{2} U_{2} \\
\quad \vdots \\
Z_{n}=\alpha_{n 1} F_{2}+\alpha_{n 2} F_{2}+\cdots+\alpha_{n n} F_{n}+c_{n} U_{n}
\end{array}\right.
$$

$Z$ : the new variables form the linear combination of initial indicators;

$F$ : common factor of initial indicators; 
Table 1. The spatial autocorrelation coefficient among the growth poles in the Yangtze River delta.

\begin{tabular}{cc}
\hline Year & Spatial Autocorrelation Coefficient \\
\hline 2007 & -0.3759 \\
2008 & -0.3307 \\
2009 & -0.3496 \\
2010 & -0.2608 \\
2011 & -0.1547 \\
\hline
\end{tabular}

Table 2. The index system measuring the level of coordinated development of regional economy.

\begin{tabular}{|c|c|c|}
\hline \multirow{26}{*}{$\begin{array}{l}\text { The level of } \\
\text { coordinated } \\
\text { development }\end{array}$} & \multirow{11}{*}{ Economic (A) } & A1, GDP growth rate (\%) \\
\hline & & A2, GDP per person \\
\hline & & A3, The proportion of tertiary industry takes in GDP (\%) \\
\hline & & A4, Revenue (ten thousand Yuan) \\
\hline & & A5, Total retail sales of social consumer goods (hundred million Yuan) \\
\hline & & A6, CPI \\
\hline & & A7, Total fixed asset investment (hundred million Yuan) \\
\hline & & A8, Real estate development investment (hundred million Yuan) \\
\hline & & A9, Savings and loans of financial institutes \\
\hline & & A10, import and export value ( ten thousand Dollar) \\
\hline & & A11, The actual amount of the utilization of foreign capital (ten thousand Dollar) \\
\hline & \multirow{9}{*}{ Social (B) } & B1, Population \\
\hline & & B2, quality of population (\%) \\
\hline & & B3, Per capita disposable income of urban residents (Yuan) \\
\hline & & B4, The per capita net income of rural households (Yuan) \\
\hline & & B5, The proportion that public services take in revenue expenditure (\%) \\
\hline & & B6, The proportion that education takes in revenue expenditure (\%) \\
\hline & & B7, The proportion that medical care takes in revenue expenditure (\%) \\
\hline & & B8, The proportion that science and technology takes in revenue expenditure (\%) \\
\hline & & B9, The proportion that social security takes in revenue expenditure (\%) \\
\hline & \multirow{6}{*}{ Environmental (C) } & C1, The total amount of Industrial wastewater discharge (ten thousand Ton) \\
\hline & & C2, The total amount of Industrial waste air discharge (standard hundred million cubic meters) \\
\hline & & C3, The total amount of Industrial dust discharge (ton) \\
\hline & & C4, The total amount of Industrial solid waste discharge (ten thousand ton) \\
\hline & & C5, The per capita green area (square meters) \\
\hline & & C6, Large-scale industrial energy consumption per GDP \\
\hline
\end{tabular}

$\alpha$ : common factor loading matrix;

$c U$ : character factor of initial indicators;

c: diagonal matrix;

The determination of $F$ will be in line with the principle of max accumulative contribution. Regard of any $Z_{i}$, the variance is as follows:

$$
\operatorname{VAR}\left(Z_{i}\right)=1 \Rightarrow \alpha_{i 1}^{2}+\alpha_{i 2}^{2}+\alpha_{i 3}^{2}+\cdots+\alpha_{i m}^{2}+\operatorname{VAR}\left(e_{i}\right)=1
$$

In the variance, $\alpha_{i 1}^{2}+\alpha_{i 2}^{2}+\alpha_{i 3}^{2}+\cdots+\alpha_{i m}^{2}$ means common variance. Larger common variance undertakes 
more information of the initial indexes. And in the factor analysis, the principal component will be selected according to the following too standards, namely the eigenvalues should be greater than 1 and the accumulative variance should be greater than $85 \%$. The concrete process includes the following three steps:

1) Do the factor analysis for the economic development level (F1), social development level (F2), and environmental development level (F3), hence determine the principal component and eigenvalues.

2) Calculate the evaluation value.

$$
F_{i}=\sum_{j=1}^{n} \alpha_{i j} y_{i j} \quad(i=1,2,3)
$$

$F_{i}$ : the evaluation value of the second level indexes, i represents for the level of economic $(i=1)$, social $(i=2)$ and environment $(i=3)$ index;

$y_{i j}$ : The third level indexes of every growth pole;

$\alpha_{i j}$ : Component matrix load.

Determine the principal component weights $W_{i j}$ for each secondary index.

For every third level index, the weight of the index is measured by the proportion that each principal component eigenvalues weight in all the component eigenvalues.

$$
W_{i j}=\frac{\lambda_{1}}{\sum_{k=1}^{j} \lambda_{k}}
$$

3) Determine the weight of the three secondary indexes by using AHP analysis, and calculate the weighted average in order to get the final score and sequence of the 16 cities.

\subsection{Data Source and Process}

The data is based on the statistic yearbooks and websites of Shanghai, Jiangsu and Zhejiang province, including the primary statistics of 26 indexes (economy, society and environment) of the 16 cities in the year 2011. Ensure that the data entering the factor analysis is in the same dimension and direction.

1) Dimensioning Process.

Undergo the dimension process by making use of the formula $X_{i j}=\left(x_{i j}-\overline{x_{j}}\right) / \sigma_{j}$ in order to unify the unit of data.

2) Direction Unifying Process.

As for most of the 26 third level indexes, positive value means better performance. As a result, considering the convenience of the calculation process, this paper chooses the forward direction as the basis direction. For those backward direction processes, this paper takes the reciprocal form of the data. As for the moderately indicators, this paper select a moderate value to undergo the direction unifying process using the formula:

$$
1 / \mid X_{i j}^{\prime} \text { - moderate value } \mid
$$

\section{The Empirical Analysis of the Level of Coordinated Development}

\subsection{KMO (Kaiser-Meyer-Olkin) and Bartlett Tests}

The KMO (Kaiser-Meyer-Olkin) and Bartlett tests are used to testify whether the database is appropriate for the factor analysis, and whether the results of the empirical tests could pass the significant test. In this empirical analysis, the KMO values of the level of economic, social and environmental development are between 0.5 and 0.6. The significance of the Bartlett tests are $0.000,0.000$ and 0.001 , which are all smaller than 0.05 . Hence, the database taken into the factor analysis is appropriate and the analysis will produce an effective result (Table 3 and Table 4).

\subsection{Determine the Principal Component of the Secondary Indexes}

The selection of the principal component abides by the principle that the eigenvalues should greater than 1 or the accumulative variation should greater than $85 \%$. Take the index of economic development into consideration. 
Table 3. The empirical results of the KMO and Bartlett tests.

\begin{tabular}{ccc}
\hline & KMO value & Bartlett (Sig.) \\
\hline The index of the level of the economic development & 0.582 & 0.000 \\
The index of the level of the social development & 0.536 & 0.000 \\
The index of the level of the environmental development & 0.583 & 0.001 \\
\hline
\end{tabular}

Table 4. The eigenvalues of the principal component and the variations.

\begin{tabular}{ccccc}
\hline Secondary Indexes & Principal Component & Value & Total Variation Explained\% & Cumulative Total Variation Explained \% \\
\hline \multirow{2}{*}{ Economy } & Z11 & 5.865 & 53.318 & 53.318 \\
& Z12 & 2.392 & 21.742 & 75.059 \\
Zociety & Z13 & 1.203 & 10.939 & 85.998 \\
& Z21 & 3.357 & 37.297 & 37.297 \\
\multirow{2}{*}{ Environment } & Z23 & 1.203 & 33.728 & 31.025 \\
& Z31 & 2.548 & 14.371 & 85.396 \\
& Z32 & 1.827 & 42.460 & 42.460 \\
\hline
\end{tabular}

As the eigenvalues of the top three principal components are all greater than 1 , as well as the accumulative variation reaches $85.998 \%$, this paper selects the top three principal components to represent the former indexes. Similarly, as for the social and environmental development, this paper respectively selects the top three principal components to represent the primary social related indexes and the top two principal components to represent the primary environmental related indexes.

\subsection{The Comprehensive Evaluation of the Secondary Indexes}

Calculate the weight average to define the comprehensive evaluation of the secondary indexes $F_{i}(I=1,2,3)$

$$
\begin{aligned}
& F_{1}=W_{Z 11} Z_{11}+W_{Z 12} Z_{12}+W_{Z 13} Z_{13} \\
& F_{2}=W_{Z 21} Z_{21}+W_{Z 22} Z_{22}+W_{Z 23} Z_{23} \\
& F_{3}=W_{Z 31} Z_{31}+W_{Z 32} Z_{32}
\end{aligned}
$$

The determination of the weight in the above formula is as follows:

$$
\begin{gathered}
\left\{\begin{array}{l}
W_{Z 11}=5.865 /(5.865+2.392+1.203)=0.6199 \\
W_{Z 12}=2.392 /(5.865+2.392+1.203)=0.2528 \\
W_{Z 13}=1.203 /(5.865+2.392+1.203)=0.1271
\end{array}\right. \\
\left\{\begin{array}{l}
W_{Z 21}=3.357 /(3.357+3.036+1.203)=0.4419 \\
W_{Z 22}=2.392 /(5.865+2.392+1.203)=0.3149 \\
W_{Z 23}=1.203 /(5.865+2.392+1.203)=0.1583
\end{array}\right. \\
\left\{\begin{array}{l}
W_{Z 31}=2.548 /(2.548+1.827)=0.5824 \\
W_{Z 32}=1.827 /(2.548+1.827)=0.4176
\end{array}\right.
\end{gathered}
$$

\subsection{The Comprehensive Evaluation of the Top Level Index}

According to the AHP methods, this paper calculates the weight among the three secondary indexes, namely $0.545,0.286$, and 0.167 . Hence the value of the top level index could be got from the formula below (Figure 2). 


$$
V=0.545 F_{1}+0.286 F_{2}+0.167 F_{3}
$$

\subsection{The Judgment of the Level of Coordinated Development}

Through measuring the Skewness and Kurtosis of the evaluation value of each city growth pole under every index, this paper could make judgment for the level of coordinated development (Table 5).

1) The judgment of the level of economic development.

The Skewness of the economic development is 1.821 , which is skewed to the right side. Such right side Skewness shows that the level of most cities' economic development is lower or slightly higher than the average 0.09031831. Only Shanghai, Suzhou, Hangzhou, Wuxi and Nanjing are higher than the average. On the other side, the Kurtosis of the economic development is 3.618, which is far higher than normal distribution. The steeple shape indicates most cities gather at the average point. From Hangzhou (2.934676239) till the end of the sequence, the number gather seriously round the range $0.09031831 \pm 2.500$, which shows that the economic development gap is comparatively large between Shanghai, Suzhou and the others.

2) The judgment of the level of social development.

The Skewness of the social development is -0.096 , which is skewed to the left side. Such left side Skewness shows that the level of most cities' social development is higher or slightly lower than the average -0.0000181 . The score of Changzhou, Yangzhou, Nantong and Taizhou $(\mathrm{J})$ is relatively lower, locating on the left tail. The shape of the social development is basically consistent with the normal distribution. Considering the social

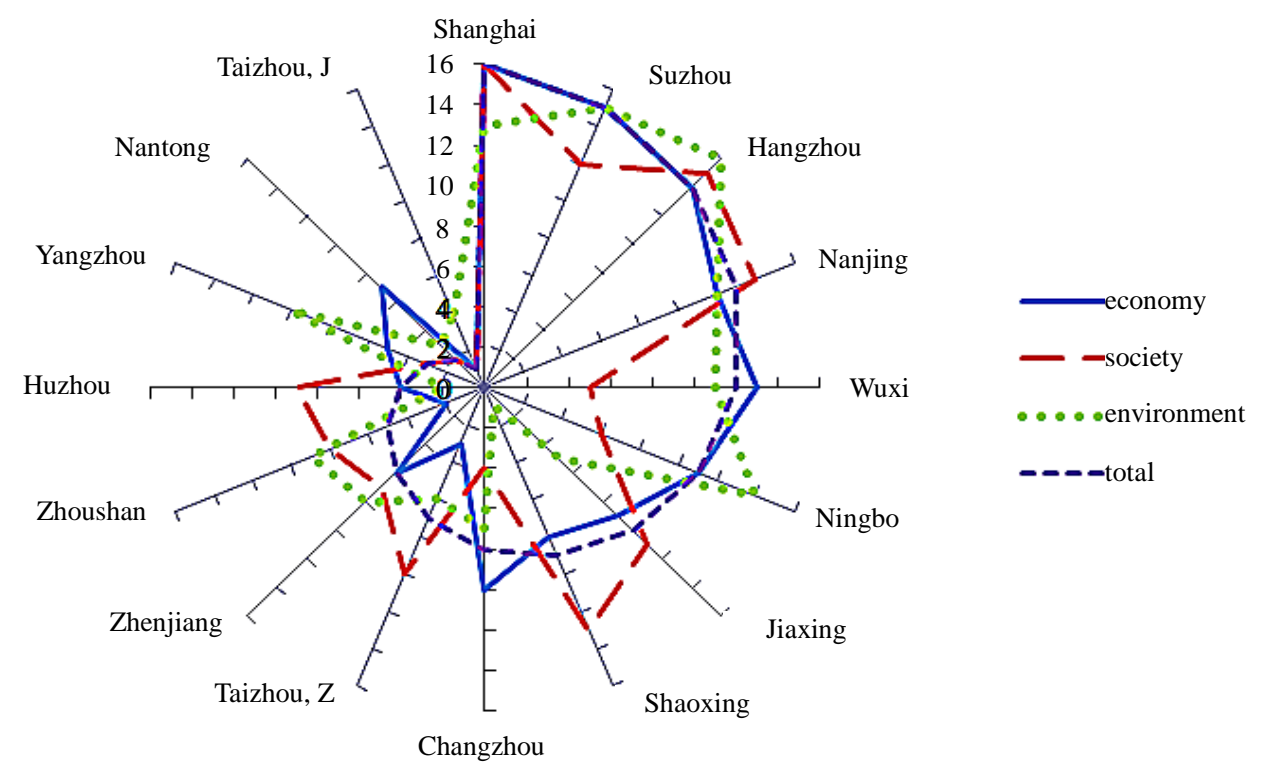

Figure 2. Ranks of the 16 growth poles in terms of economy, society, environment and total judgments.

Table 5. Statistical description of the evaluation results.

\begin{tabular}{|c|c|c|c|c|c|c|c|c|c|}
\hline & \multirow{2}{*}{ Min } & \multirow{2}{*}{ Max } & \multirow{2}{*}{ Average } & \multirow{2}{*}{ Standard Error } & \multirow{2}{*}{ Variation } & \multicolumn{2}{|c|}{ Skewness } & \multicolumn{2}{|r|}{ Kurtosis } \\
\hline & & & & & & Value & Standard Error & Value & Standard Error \\
\hline Economy & -4.45406 & 12.88548E1 & $-9.03183 E-2$ & 4.54187 & 20.629 & 1.821 & 0.564 & 3.618 & 1.091 \\
\hline Society & -4.09469 & 4.19352 & $-1.80445 E-5$ & 2.30952 & 5.334 & -0.096 & 0.564 & -0.722 & 1.091 \\
\hline Environment & -6.78642 & 7.54447 & -1.96357 & 4.59381 & 21.103 & 0.743 & 0.564 & -0.774 & 1.091 \\
\hline Total & -4.60918 & 8.66137 & $-3.77145 \mathrm{E}-1$ & 3.51501 & 12.355 & 1.373 & 0.564 & 1.607 & 1.091 \\
\hline
\end{tabular}


development performance, the variation is less serious than the level of economic development.

3) The judgment of the level of environmental development.

The Skewness of the environmental development is 0.743 , which is skewed to the right side. The score of Hangzhou, Suzhou, Ningbo, Shanghai and Nanjing is higher than any other cities, which is located in the right tail of the shape. The score of other 11cities is relatively close to each other, which is located in the left zone of the shape. Although a minority of cities' score is higher than other cities, the part that overwhelms the average level does not influence too much toward the variation of the development.

4) The judgment of the comprehensive index.

The Skewness of the comprehensive index is 1.373, which is skewed to the right side. The score of Shanghai, Suzhou, Hangzhou, Nanjing, Wuxi and Ningbo is higher than any other cities, which is located in the right tail of the shape. The score of other 10cities is relatively close to each other, which is located in the left zone of the shape. On the other side, the Kurtosis of the comprehensive index is 1.607 , which is slightly higher than normal distribution. The Kurtosis of the comprehensive index is smaller than the Kurtosis of economic development, which shows that the agglomeration of the comprehensive index is lower than the Kurtosis of economic development.

In conclusion, the Skewness and Kurtosis value of the comprehensive index is in line with the economic ones which are the same as the sequence of the 16 cities in both the comprehensive and economic column. However, as the existence of the variation of the social and environmental elements, the Skewness and Kurtosis value of the comprehensive index is not as much higher than the economic effects. All the results of the empirical analysis show that the economic variation is the main key to the agglomeration effect. On the contrary, the less serious variation of the social and environmental is benefit for the weakening of the agglomeration effect, which will do good to the regional coordinated development.

\section{Discussion}

It is concluded that, in the Yangtze River Delta, there exists the agglomeration effect. At the same time, merits also help to improve the coordinated development of the regional economy.

1) The growth poles system of the Yangtze River Delta has taken a frame of "one center with two sub ones" into consideration. The cities of Shanghai, Nanjing and Hangzhou have formed a strong ability to gravitate. And in order to seek for the regional coordinated development, the other cities should insist dislocation development to hunt for potential development and growth opportunities.

The individualization process of the urban development should focus on the variation of the function and industry and make full use of the resource basement and historical development precipitation. Firstly, based on the development edge of each city or urban agglomeration, different growth poles should clarify its role to play in this region. For example, with the role of international trade, shipping and finance center that shanghai takes, the southern part of Jiangsu area could actively participate to develop its manufacturing and inner transportation industry, cultivating the Nanjing agglomeration and the integration of Nanjing, Yangzhou and Zhenjiang city to work with Suzhou, Wuxi, and Changzhou agglomeration.

The differentiation of the industrial development is the direct reflection of the individualization process of the urban development. Each city should select its own leading industries and inhibit the vicious competition and structure similarity. In the mature market, each city should seek for the market differentiations to obtain the competitive edge.

2) The government can promote the integration of infrastructure among cities to effectively support the circulation of elements among cities so as to further promote the diffusion effect of the growth poles. The key to build up social and environmental infrastructure is to ensure the sharing and communication mechanism, and to increase the level of transportation, energy, information, social affairs and public service integration, hence lowering the transportation cost and viscous characteristics of the administrative management. At the same time, by laws and administrate regulations, the government should promote the quality of living environment in medium and small cities, protect the unique of small ecological environment, encourage a free flow of resources from metropolis to medium and small cities and towns to raise the level of coordinated development in the Yangtze River Delta.

3) The cities in the Yangtze River Delta should instantly put the problem of eco-protection into the frame of coordinated development, and pay enough attention to the environmental protection from the following two as- 
pects, namely the manufacturing of enterprises and living conditions of local citizens. The environmental problems caused by the local residents could be prevented or solved by media campaigns; however, the environmental problems caused by the enterprises should otherwise need the corporation among cities and the relative administrative regulations. Take the blue-green algae as the example, although the main polluted spot is in Wuxi, however, considering the cultivate management in both the upstream and downstream; all the cities around the Tai Lake should get involved. As a result, in teams of environmental protection, the region itself should establish rewards and punishments systems and relative laws and regulations, and furthermore, to make all the documents effective to all the cities in the region.

\section{References}

[1] Perroux, F. (1989) The Concept of Growth Poles. Renditions of Economics, 9, 112-156.

[2] Yang, D.L. (1995) Changes of China’s Inter Provincial Variation since Reform. China Industrial Economy, 1, $24-30$.

[3] Pu, Y.X., Ge, Y., Ma, R.H., Huang, X.Y. and Ma, X.D. (2005) Analyzing Regional Economic Disparities Based on ESDA. Geographical Research, 11, 107-112.

[4] Liu, S.H. (2001) The Application of the Growth Pole Theory in China. China Economist, 8, 53-61.

[5] He, Y.L. and Zhang, R.Z. (2007) An Analysis of the Point, Axis and Cluster Theory. Journal of Lanzhou University, 7, 11-18.

[6] Zhang, Z.T. (2009) The Achievement of the Diffusion Effect of the Growth Pole. Jiangsu Social Sciences, 11, 121125.

[7] Zhu, J.C. (2011) The Yangtze River Delta Coordination Development Based on Polycentric and Regional Symbiosis. Population, Resources and Environment, 3, 112-123.

[8] Deng, X.R. and Liu, X. (2007) The Comparative Research of the Innovation Agglomeration and Diffusion Effects Between the Bohai Rim and the Yangtze River Delta. Forum on Science and Technology in China, 11, 71-80. 